Préface

Les multiples besoins de la société moderne, basée sur la technologie, nécessiten
d'effectuer toute sorte de mesures. Leurs domaines d'applications vont des d'effectuer toute sorte de mesures. Leurs domaines d applications vont des expé-

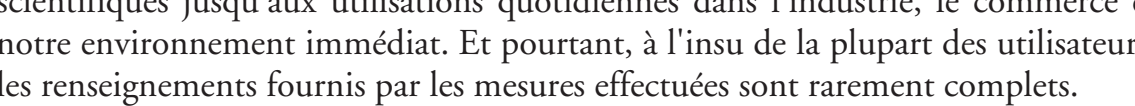
En effet, tout résultat de mesure n'est en général qu’une estimation de la valeur de la quantité mesurée, la «vraie » valeur reste inconnue. Pour cette raison, on devrait
évaluer la dispersion des valeurs de cette "valeur estimée " qui pourrait être attriEn terme de métrologie, la mesure de ce paramètre de dispersion est appelée l'incertitude de mesure. Lorsque l'utilisateur connaît la valeur de ce paramètre, il peut éva-
luer la confiance dans le résultat de la mesure effectuée. Malheureusement, n'existe pas de moyen unique et universel d'exprimer quantitativement le « doute
que représente l'incertitude. Cette situation a conduit à l'élaboration de différentes Pour palier ce manque de commun accord, en 1978, le Comité International des
Poids et Mesures (CIPM) a demandé à son organe exécutiff, le Bureau international

iii 
des Poids et Mesures (BIPM), de formuler des principes fondamentaux de l'évaluation de l'incertitude, ce qui l'a conduit à élaborer la Recommandation INC-1 (1980).

Un document détaillant les méthodes destinées à mettre en œuvre ces principes a ensuite été élaboré. Ce document appelé GUM « Guide pour l'expression de l'incertitude de mesure » en anglais " Guide to the expression of Uncertainty in Measurement », est publié par l'ISO au nom du BIPM, de la CEI, de l'OIML (Organisation Internationale de la Métrologie Légale), de la Fédération Internationale de Chimie Clinique (IFCC), de l'Union Internationale de Chimie Pure et Appliquée (UICPA) et l'Union Internationale de Physique Pure et Appliquée (UIPPA). Ce document faisant autorité est populairement connu sous le nom de GUM et ce sont les règles prescrites dans ce GUM qui sont utilisées dans le présent ouvrage.

Le présent ouvrage fait partie d'une série de deux livres. Le premier a pour but d'illustrer les méthodes pratiques permettant d'estimer les incertitudes de mesure dans les domaines du pesage, des mesures de température et des mesures dimensionnelles.

Quant au second, il présente des applications concrètes pour l'estimation des incertitudes dans les essais et les analyses.

Le problème de l'exécution d'un budget d'incertitude est en fait assez simple dans la plupart des cas. Sa solution ne représente que la formulation d'un modèle de mesure approprié, et à l'évaluation de ce modèle, conformément aux règles données dans le GUM. Néanmoins, mon expérience tant au sein d'un laboratoire national qu'auprès des laboratoires d'étalonnage accrédités ou industriels m'a montré que, lorsqu'ils sont confrontés à la tâche d'estimer les incertitudes, la plupart des praticiens pensent qu'ils entrent en territoire inexploré. C'est très probablement dû au fait que, comme son titre l'indique, le GUM n'est pas un manuel. Il s'agit d'un document plutôt laconique. Le contenu du texte est condensé et ne s'attarde pas beaucoup sur les aspects théoriques, se concentrant plutôt sur la fourniture d'un ensemble de règles qui couvrent bien la plupart des situations de mesure susceptibles d'être rencontrées dans la pratique.

Ce livre est donc une tentative pour répondre aux besoins d'un très large public. Il devrait convenir aux métrologues des laboratoires d'étalonnage et des laboratoires industriels qui souhaitent développer leurs connaissances de l'estimation des incertitudes. Il devrait également être utile aux enseignants et aux étudiants pour les cours sur la qualité, la métrologie, l'instrumentation et les sujets connexes au niveau des universités, des écoles d'ingénieurs et des instituts techniques. Il devrait aussi présenter beaucoup d'intérêt aux étudiants diplômés effectuant des recherches expérimentales dans tous les domaines de la science et la technologie. Il devrait également convenir à des scientifiques et des ingénieurs engagés dans la recherche et le développement. 
Georges BONNIER est expert international en métrologie. Ex-directeur adjoint de l'Institut National de Métrologie (Désormais LNE/INM/CNAM), il est expert technique en métrologie des températures. Aujourd'hui il exerce une activité de conseiller scientifique et technique auprès du Comité Africain de Métrologie (CAFMET, http://www.acmetrology.com). 


\section{This page intentionally left blank}

